Article

\title{
Analysis of Hilfer Fractional Integro-Differential Equations with Almost Sectorial Operators
}

\author{
Kulandhaivel Karthikeyan ${ }^{1}(\mathbb{D})$, Amar Debbouche ${ }^{2,3, *(D)}$ and Delfim F. M. Torres ${ }^{3}$ (D) \\ 1 Department of Mathematics, Centre for Research and Development, KPR Institute of Engineering and \\ Technology, Coimbatore, Tamil Nadu 641407, India; karthi_phd2010@yahoo.co.in \\ 2 Department of Mathematics, Guelma University, Guelma 24000, Algeria \\ 3 Center for Research and Development in Mathematics and Applications (CIDMA), Department of \\ Mathematics, University of Aveiro, 3810-193 Aveiro, Portugal; delfim@ua.pt \\ * Correspondence: amar_debbouche@yahoo.fr
}

\section{check for}

updates

Citation: Karthikeyan, K.; Debbouche, A.; Torres, D.F.M. Analysis of Hilfer Fractional Integro-Differential Equations with Almost Sectorial Operators. Fractal Fract. 2021, 5, 22. https://doi.org/ $10.3390 /$ fractalfract 5010022

Received: 31 December 2020

Accepted: 4 March 2021

Published: 8 March 2021

Publisher's Note: MDPI stays neutral with regard to jurisdictional claims in published maps and institutional affiliations.

\begin{abstract}
In this work, we investigate a class of nonlocal integro-differential equations involving Hilfer fractional derivatives and almost sectorial operators. We prove our results by applying Schauder's fixed point technique. Moreover, we show the fundamental properties of the representation of the solution by discussing two cases related to the associated semigroup. For that, we consider compactness and noncompactness properties, respectively. Furthermore, an example is given to illustrate the obtained theory.
\end{abstract}

Keywords: Hilfer fractional derivatives; mild solutions; almost sectorial operators; measure of non-compactness

MSC: 26A33; 47B12

\section{Introduction}

We consider nonlocal integro-differential equations involving Hilfer fractional derivatives and almost sectorial operators:

$$
\begin{gathered}
D_{0^{+}}^{\alpha, \gamma} u(t)+\mathcal{A} u(t)=g\left(t, u(t), \int_{0}^{t} k(t, s) f(s, u(s)) d s\right), \quad t \in(0, T]=\mathcal{J}, \\
\left.I_{0^{+}}^{(1-\alpha)(1-\gamma)}[u(t)]\right|_{t=0}+h(u(t))=u_{0},
\end{gathered}
$$

where $D_{0^{+}}^{\alpha, \gamma}$ is the Hilfer fractional derivative of order $\alpha \in(0,1)$ and type $\gamma \in[0,1]$. We assume that $\mathcal{A}$ is an almost sectorial operator on a Banach space $\mathcal{Y}$ with norm $\|\cdot\|$. Let $f: \mathcal{J} \times \mathcal{Y} \rightarrow \mathcal{Y}, g: \mathcal{J} \times \mathcal{Y} \times \mathcal{Y} \rightarrow \mathcal{Y}$ and $h: C(\mathcal{J}: \mathcal{Y}) \rightarrow \mathcal{Y}$ to be given abstract functions to be specified later. For brevity, we take

$$
\mathcal{B} u(t)=\int_{0}^{t} k(t, s) f(s, u(s)) d s .
$$

Over the last decades, mathematical modeling has been supported by the field of fractional calculus, with several successful results and fractional operators shown to be an excellent tool to describe the hereditary properties of various materials and processes. Recently, this combination has gained a large amount of importance, mainly because fractional differential equations have become powerful tools for the modeling of several complex phenomena in numerous seemingly diverse and widespread fields of science and engineering; see, for instance, the basic text books in [1-4] and recent research works in [5-7]. In fact, abrupt changes, such as shocks, harvesting, or natural disasters, may occur in the dynamics of evolving processes. These short-term perturbations are often treated 
in the form of impulses. Recently, in many published works, Hilfer fractional differential equations have received attention [8-17].

In [15], Jaiswal and Bahuguna studied equations of Hilfer fractional derivatives with almost sectorial operators in the abstract sense:

$$
\begin{aligned}
D_{0^{+}}^{\lambda, v} u(t)+\mathcal{A} u(t) & =g(t, u(t)), \quad t \in(0, T], \\
\mathcal{I}_{0^{+}}^{(1-\lambda)(1-v)} u(0) & =u_{0} .
\end{aligned}
$$

We also refer to the work in [8], where Ahmed et al. studied the question of the existence of nonlinear Hilfer fractional differential equations with controls. Sufficient conditions are also established, where the time fractional derivative is the Hilfer derivative. In [18], Zhang and Zhou studied fractional Cauchy problems with almost sectorial operators of the form

$$
\begin{aligned}
& \left({ }^{L} D_{0+}^{q} x\right)(t)=A x(t)+f(t, x(t)), \text { for almost all } t \in[0, a], \\
& \left(I_{0^{+}}^{(1-q)} x\right)(0)=x_{0}
\end{aligned}
$$

where ${ }^{L} D_{0+}^{q}$ is the Riemann-Liouville derivative of order $q, I_{0^{+}}^{(1-q)}$ is the Riemann-Liouville integral of order $1-q, 0<q<1, A$ is an almost sectorial operator on a complex Banach space, and $f$ is a given function. Motivated by these results, here, we extend the previous available results of the literature to a class of Hilfer fractional integro-differential equations in which the closed operator is almost sectorial. Moreover, we also consider both compactness and noncompactness cases of the semigroup operator.

The paper is structured as follows. In Section 2, we present necessary information about the Hilfer derivative, almost sectorial operators, measures of non-compactness, mild solutions of Equations (1) and (2) and some useful definitions, results and lemmas. We discuss fundamental results for mild solutions to Equations (1) and (2) in Section 3. In Section 4, we prove the solvability question in two cases, when associated semigroups are compact and noncompact, respectively. An example is then given in Section 5 to illustrate our main results. We end with Section 6, which presents our conclusions.

\section{Preliminaries}

In this section, we recall the necessary theory that is used throughout the work in order to obtain new results.

\subsection{Fractional Derivatives}

We start with a short introduction of the main definitions in fractional calculus $[13,19]$.

Definition 1. The left-sided Riemann-Liouville fractional integral of order $\alpha>0$ with a lower limit a for a function $h:[a,+\infty) \rightarrow \mathbb{R}$ is defined as

$$
I_{a^{+}}^{\alpha} h(t)=\frac{1}{\Gamma(\alpha)} \int_{a}^{t}(t-s)^{\alpha-1} h(s) d s,
$$

provided the right hand side is defined almost everywhere (a.e.) on $[a,+\infty)$.

Remark 1. If $a=0$, then we write $I_{0^{+}}^{\alpha} f(t)=\left(g_{\alpha} * f\right)(t)$, where

$$
g_{\alpha}(t):= \begin{cases}\frac{1}{\Gamma(\alpha)} t^{\alpha-1}, & t>0 \\ 0, & t \leq 0\end{cases}
$$

and, as usual, $*$ denotes the convolution of functions. Note that $\lim _{\alpha \rightarrow 0^{+}} g_{\alpha}(t)=\delta(t)$ with $\delta$ the delta Dirac function. 
Definition 2. The left-sided Riemann-Liouville fractional derivative of order $\alpha>0, n-1 \leq \alpha<$ $n, n \in \mathbb{N}$, for a function $h:[a,+\infty) \rightarrow \mathbb{R}$, is defined by

$$
{ }^{L} D_{a^{+}}^{\alpha} h(t)=\frac{1}{\Gamma(n-\alpha)} \frac{d^{n}}{d t^{n}} \int_{a}^{t} \frac{h(s)}{(t-s)^{\alpha+1-n}} d s, \quad t>a,
$$

provided the right hand side is defined a.e. on $[a,+\infty)$.

Definition 3. The left-sided Caputo's fractional derivative of order $\alpha>0, n-1<\alpha<n, n \in \mathbb{N}$, for a function $h:[a,+\infty) \rightarrow \mathbb{R}$, is defined by

$$
{ }^{C} D_{a^{+}}^{\alpha} h(t)=\frac{1}{\Gamma(n-\alpha)} \int_{a}^{t} \frac{h^{(n)}(s)}{(t-s)^{\alpha+1-n}} d s=I_{a^{+}}^{n-\alpha} h^{(n)}(t), \quad t>a,
$$

provided the right hand side is defined a.e. on $[a,+\infty)$.

Definition 4. The left-sided Hilfer fractional derivative of order $0<\alpha<1$ and type $\gamma \in[0,1]$, of a function $h:[a,+\infty) \rightarrow \mathbb{R}$, is defined as

$$
D_{a^{+}}^{\alpha, \gamma} h(t)=\left[I_{a^{+}}^{(1-\alpha) \gamma} D\left(I_{a^{+}}^{(1-\alpha)(1-\gamma)} h\right)\right](t)
$$

Remark 2. (i) If $\gamma=0,0<\alpha<1$ and $a=0$, then the Hilfer fractional derivative corresponds to the classical Riemann-Liouville fractional derivative:

$$
D_{0^{+}}^{\alpha, 0} h(t)=\frac{d}{d t} I_{0^{+}}^{1-\alpha} h(t)={ }^{L} D_{0^{+}}^{\alpha} h(t) .
$$

(ii) If $\gamma=1,0<\alpha<1$ and $a=0$, then the Hilfer fractional derivative corresponds to the classical Caputo fractional derivative:

$$
D_{0^{+}}^{\alpha, 1} h(t)=I_{0^{+}}^{1-\alpha} \frac{d}{d t} h(t)={ }^{C} D_{0^{+}}^{\alpha} h(t) .
$$

\subsection{Measure of Non-Compactness}

The motivation to consider our problem can be found in $[18,19]$. Here, we generalize the results in $[18,19]$. Let $\mathcal{L} \subset \mathcal{Y}$ be bounded. The Hausdorff measure of non-compactness is considered as

$$
\Theta(\mathcal{L})=\inf \left\{\theta>0 \quad \text { such that } \quad \mathcal{L} \subset \bigcup_{j=1}^{m} B_{\theta}\left(x_{j}\right), \quad \text { where } \quad x_{j} \in \mathcal{Y}, m \in N\right\}
$$

while the Kurtawoski measure of noncompactness $\Phi$ on a bounded set $\mathcal{B} \subset \mathcal{Y}$ is given by

$$
\Phi(\mathcal{L})=\inf \left\{\epsilon>0 \quad \text { such that } \quad \mathcal{L} \subset \bigcup_{j=1}^{m} M_{j} \text { and } \operatorname{diam}\left(M_{j}\right) \leq \epsilon\right\}
$$

with the following properties:

1. $\mathcal{L}_{1} \subset \mathcal{L}_{2}$ gives $\Theta\left(\mathcal{L}_{1}\right) \leq \Theta\left(\mathcal{L}_{2}\right)$, where $\mathcal{L}_{1}, \mathcal{L}_{2}$ are bounded subsets of $\mathcal{Y}$;

2. $\Theta(\mathcal{L})=0$ if and only if $\mathcal{L}$ is relatively compact in $\mathcal{Y}$;

3. $\Theta(\{z\} \cup \mathcal{L})=\Theta(\mathcal{L})$ for all $z \in \mathcal{Y} \mathcal{L} \subseteq \mathcal{Y}$;

4. $\Theta\left(\mathcal{L}_{1} \cup \mathcal{L}_{2}\right) \leq \max \left\{\Theta\left(\mathcal{L}_{1}\right), \Theta\left(\mathcal{L}_{2}\right)\right\}$;

5. $\Theta\left(\mathcal{L}_{1}+\mathcal{L}_{2}\right) \leq \Theta\left(\mathcal{L}_{1}\right)+\Theta\left(\mathcal{L}_{2}\right)$;

6. $\Theta(r \mathcal{L}) \leq|r| \Theta(\mathcal{L})$ for $r \in \mathbb{R}$. 
Let $\mathcal{M} \subset C(I, \mathcal{Y})$ and $\mathcal{M}(r)=\{v(r) \in \mathcal{Y} \mid v \in \mathcal{M}\}$. One defines

$$
\int_{0}^{t} \mathcal{M}(r) d r:=\left\{\int_{0}^{t} v(r) d r \mid v \in \mathcal{M}\right\}, \quad t \in \mathcal{J} .
$$

Proposition 1. If $\mathcal{M} \subset C(\mathcal{J}, \mathcal{Y})$ is equicontinuous and bounded, then $t \rightarrow \Theta(\mathcal{M}(t))$ is continuous on I. Furthermore,

$$
\Theta(\mathcal{M})=\max \left\{\Theta(\mathcal{M}(t)), \Theta\left(\int_{0}^{t} v(r) d r\right)\right\} \leq \int_{0}^{t} \Theta(v(r)) d r, \quad \text { for } \quad t \in I .
$$

Proposition 2. Let $\left\{v_{n}: \mathcal{J} \rightarrow \mathcal{Y}, n \in \mathbb{N}\right\}$ be Bochner integrable functions. This implies that $\left\|v_{n}\right\| \leq m(t)$ a.e. for $n \in \mathbb{N}$ and $m \in L^{1}\left(I, R^{+}\right)$. Then, $\xi(t)=\Theta\left(\left\{v_{n}(t)\right\}_{n=1}^{\infty}\right) \in L^{1}\left(I, R^{+}\right)$ and satisfies

$$
\Theta\left(\left\{\int_{0}^{t} v_{n}(r) d r: n \in \mathbb{N}\right\}\right) \leq 2 \int_{0}^{t} \xi(r) d r
$$

Proposition 3. Let $\mathcal{M}$ be a bounded set. Then, for any $\theta>0$, there exists a sequence $\left\{v_{n}\right\}_{n=1}^{\infty} \subset$ $\mathcal{M}$ such that

$$
\Theta(\mathcal{M}) \leq 2 \Theta\left\{v_{n}\right\}_{n=1}^{\infty}+\theta
$$

\subsection{Almost Sectorial Operators}

Let $0<\beta<\pi$ and $-1<\beta<0$. We define $S_{\beta}^{0}:=\{v \in C \backslash\{0\}$ such that $|\arg v|<\beta\}$ and its closure by $S_{\beta}$, such that $S_{\beta}=\{v \in C \backslash\{0\}$ with $|\arg v| \leq \beta\} \cup\{0\}$.

Definition 5 (See [20]). For $-1<\beta<0$ and $0<\omega<\frac{\pi}{2}$, we define $\left\{\Theta_{\omega}^{\beta}\right\}$ as the family of all closed and linear operators $\mathcal{A}: D(\mathcal{A}) \subset \mathcal{Y} \rightarrow \mathcal{Y}$ such that

1. $\sigma(\mathcal{A})$ is contained in $S_{\omega}$;

2. for all $\beta \in(\omega, \pi)$ there exists $M_{\beta}$ such that

$$
\|\mathcal{R}(z, \mathcal{A})\|_{L(\mathcal{Y})} \leq M_{\beta}|z|^{\beta}
$$

where $\mathcal{R}(z, \mathcal{A})=(z I-\mathcal{A})^{-1}$ is the resolvent operator and $\mathcal{A} \in \Theta_{\omega}^{\beta}$ is said to be an almost sectorial operator on $\mathcal{Y}$.

Proposition 4 (See [20]). Let $\mathcal{A} \in \Theta_{\omega}^{\beta}$ for $-1<\beta<0$ and $0<\omega<\frac{\pi}{2}$. Then the following properties hold:

1. $Q(t)$ is analytic and $\frac{d^{n}}{d t^{n}} Q(t)=\left(-\mathcal{A}^{n} Q(t)\left(t \in S_{\frac{\pi}{2}}^{0}\right)\right.$;

2. $Q(t+s)=Q(t) Q(s) \forall t, s \in S_{\frac{\pi}{2}}^{0}$;

3. $\|Q(t)\|_{L(\mathcal{Y})} \leq C_{0} t^{-\beta-1}(t>0)$, where $C_{0}=C_{0}(\beta)>0$ is a constant;

4. if $\sum_{Q}=\left\{x \in \mathcal{Y}: \lim _{t \rightarrow 0_{+}} Q(t) x=x\right\}$, then $D\left(\mathcal{A}^{\theta}\right) \subset \sum_{Q}$ if $\theta>1+\beta$;

5. $\mathcal{R}(r,-\mathcal{A})=\int_{0}^{\infty} e^{-r s} Q(s) d s, r \in \mathbb{C}$ with $\operatorname{Re}(r)>0$.

We use the following Wright-type function [19]:

$$
M_{\alpha}(\theta)=\sum_{n \in \mathbb{N}} \frac{(-\theta)^{n-1}}{\Gamma(1-\alpha n)(n-1) !}, \quad \theta \in \mathbb{C} .
$$

For $-1<\sigma<\infty, r>0$, the following properties hold:

(A1) $M_{\alpha}(\theta) \geq 0, t>0$;

(A2) $\int_{0}^{\infty} \theta^{\sigma} M_{\alpha} d \theta=\frac{\Gamma(1+\sigma)}{\Gamma(1+\alpha \sigma)}$; 
(A3) $\int_{0}^{\infty} \frac{\alpha}{\theta^{\alpha+1}} e^{-r \theta} M_{\alpha}\left(\frac{1}{\theta^{\alpha}}\right) d \theta=e^{-r^{\alpha}}$.

The characteristic operators $\left.\left\{S_{\alpha}(t)\right\}\right|_{t \in S_{\frac{\pi}{2}-w}^{0}}$ and $\left.\left\{T_{\alpha}(t)\right\}\right|_{t \in S_{\frac{\pi}{2}-w}^{0}}$ are defined by

$$
S_{\alpha}(t):=\int_{0}^{\infty} M_{\alpha}(\theta) Q\left(t^{\alpha} \theta\right) d \theta
$$

and

$$
T_{\alpha}(t):=\int_{0}^{\infty} \alpha \theta M_{\alpha}(\theta) Q\left(t^{\alpha} \theta\right) d \theta
$$

Theorem 1 (See Theorem 4.6.1 of [19]). For each fixed $t \in S_{\frac{\pi}{2}-\omega^{\prime}}^{0} S_{\alpha}(t)$ and $T_{\alpha}(t)$ are bounded linear operators on $\mathcal{Y}$. Moreover,

$$
\left\|S_{\alpha}(t)\right\| \leq C_{1} t^{-\alpha(1+\beta)}, \quad\left\|T_{\alpha}(t)\right\| \leq C_{2} t^{-\alpha(1+\beta)}, \quad t>0,
$$

where $C_{1}$ and $C_{2}$ are constants dependent on $\alpha$ and $\beta$.

Theorem 2 (See [19]). The operators $S_{\alpha}(t)$ and $T_{\alpha}(t)$ are continuous in the uniform operator topology for $t>0$. For $s>0$, the continuity is uniform on $[s, \infty]$.

Define $\Omega_{r}(\mathcal{J}):=\{y \in C(\mathcal{J}, \mathcal{Y}) \mid\|y\| \leq r\}$. Our main results are proved in relation to the following hypotheses:

Hypothesis 1 (H1). For $t \in \mathcal{J}, g(t, \cdot, \cdot): \mathcal{Y} \times \mathcal{Y} \rightarrow \mathcal{Y}$ and $f(t, \cdot): \mathcal{Y} \rightarrow \mathcal{Y}$ are continuous functions and, for each $u \in C(\mathcal{J}, \mathcal{Y}), g(\cdot, u, \mathcal{B} u): \mathcal{J} \rightarrow \mathcal{Y}$ and $f(\cdot, u): \mathcal{J} \rightarrow \mathcal{Y}$ are strongly measurable.

Hypothesis 2 (H2). There exist functions $k_{1}, k_{2} \in L^{1}\left(\mathcal{J}, \mathbb{R}^{+}\right)$satisfying $\|g(t, \cdot, \cdot)\| \leq k_{1}(t)+$ $k_{2}(t) e^{-\delta t}$ for all $u \in \Omega_{r}(\mathcal{J})$ and almost all $t$ on $\mathcal{J}$ and

$$
I_{0^{+}}^{-\alpha \beta}\left[k_{1}(t)+k_{2}(t) e^{-\delta t}\right] \in C(\mathcal{J}, \mathbb{R}), \lim _{t \rightarrow 0^{+}} t^{(1+\alpha \beta)(1-\gamma)} I_{0^{+}}^{-\alpha \beta}\left[k_{1}(t)+k_{2}(t) e^{-\delta t}\right]=0 .
$$

Hypothesis 3 (H3). Function $h: C(\mathcal{J}, \mathcal{Y}) \rightarrow \mathcal{Y}$ is completely continuous and there exists a positive constant $k$ such that $\|h(u)\| \leq k$.

Hypothesis 4 (H4). We assume that

$$
\begin{aligned}
& \sup _{[0, T]}\left(t^{(1+\alpha \beta)(1-\gamma)}\left\|S_{\alpha, \gamma}(t)\left[u_{0}+k\right]\right\|+t^{(1+\alpha \beta)(1-\gamma)} \int_{0}^{t}(t-r)^{-\alpha \beta-1}\left[k_{1}(r)+k_{2}(r) e^{-\delta r}\right] d r\right) \leq r, \\
& \text { for } r>0, u_{0} \in D\left(\mathcal{A}^{\theta}\right) \text {, and } \theta>1+\beta, \text { where } S_{\alpha, \gamma}(t)=I_{0^{+}}^{\gamma(1-\alpha)} t^{\alpha-1} T_{\alpha}(t) .
\end{aligned}
$$

For the next two lemmas, we refer to [9,12].

Lemma 1 (See $[9,12])$. The fractional Cauchy problem (1)-(2) is equivalent to the integral equation

$$
u(t)=\frac{\left[u_{0}-h(u(t))\right]}{\Gamma(\gamma(1-\alpha)+\alpha)} t^{(1-\alpha)(\gamma-1)}+\frac{1}{\Gamma(\alpha)} \int_{0}^{1}(t-r)^{\alpha-1}[-\mathcal{A} u(r)+g(r, u(r),(\mathcal{B} u) r)] d r, \quad t \in \mathcal{J}
$$

Lemma 2 (See $[9,12])$. If $u$ is a solution to the integral equation (3), then it satisfies

$$
u(t)=S_{\alpha, \gamma}(t)\left[u_{0}-h(u(t))\right]+\int_{0}^{t} R_{\alpha}(t-r) g(r, u(r),(\mathcal{B} u) r) d r,
$$


where $S_{\alpha, \gamma}(t)=I_{0^{+}}^{\gamma(1-\alpha)} R_{\alpha}(t)$ with $R_{\alpha}=t^{\alpha-1} T_{\alpha}(t)$.

Definition 6. By a mild solution of the Cauchy problem in Equations (1) and (2), we mean a function $u \in C(\mathcal{J}, \mathcal{Y})$ that satisfies

$$
u(t)=S_{\alpha, \gamma}(t)\left[u_{0}-h(u(t))\right]+\int_{0}^{t} R_{\alpha}(t-r) g(r, u(r),(\mathcal{B} u) r) d r, \quad t \in \mathcal{J} .
$$

We define operator $\mathcal{P}: \Omega_{r}(\mathcal{J}) \rightarrow \Omega_{r}(\mathcal{J})$ as

$$
(\mathcal{P} u)(t):=S_{\alpha, \gamma}(t)\left[u_{0}-h(u(t))\right]+\int_{0}^{t}(t-r)^{\alpha-1} T_{\alpha}(t-r) g(r, u(r),(\mathcal{B} u) r) d r .
$$

Lemma 3 (See [15]). The operators $R_{\alpha}(t)$ and $S_{\alpha, \gamma}(t)$ are bounded linear operators on $\mathcal{Y}$ for every fixed $t \in S_{\frac{\pi}{2}-\omega}^{0}$. Also, for $t>0$, we have

$$
\left\|R_{\alpha}(t) x\right\| \leq C_{2} t^{-1-\alpha \beta}\|x\|, \quad\left\|S_{\alpha, \gamma}(t) x\right\| \leq \frac{\Gamma(-\alpha \beta)}{\Gamma(\gamma(1-\alpha)-\alpha \beta)} C_{2} t^{\gamma(1-\alpha)-\alpha \beta-1}\|x\| .
$$

Proposition 5 (See [15]). The operators $R_{\alpha}(t)$ and $S_{\alpha, \gamma}(t)$ are strongly continuous for $t>0$.

\section{Auxiliary Results}

Below, we present our original contributions.

Theorem 3. Let $\mathcal{A} \in \Theta_{\omega}^{\beta}$ for $-1<\beta<0$ and $0<\omega<\frac{\pi}{2}$. Assuming that (H1)-(H4) are satisfied, then the operator $\left\{\mathcal{P} y: y \in \Omega_{r}(\mathcal{J})\right\}$ is equicontinuous, provided $u_{0} \in \mathcal{D}\left(\mathcal{A}^{\theta}\right)$ with $\theta>1+\beta$.

Proof. For $y \in \Omega_{r}(\mathcal{J})$ and $t_{1}=0<t_{2} \leq T$, we have

$$
\begin{aligned}
\| \mathcal{P} y\left(t_{2}\right)- & \mathcal{P} y(0) \| \\
= & \left\|t_{2}^{(1+\alpha \beta)(1-\gamma)}\left(S_{\alpha, \gamma}\left(t_{2}\right)\left[u_{0}-h(u(t))\right]+\int_{0}^{t_{2}}\left(t_{2}-r\right)^{\alpha-1} T_{\alpha}\left(t_{2}-r\right) g(r, u(r),(\mathcal{B} u) r) d r\right)\right\| \\
\leq & \left\|t_{2}^{(1+\alpha \beta)(1-\gamma)} S_{\alpha, \gamma}\left(t_{2}\right)\right\|\left(u_{0}+k\right) \\
& +\left\|t_{2}^{(1+\alpha \beta)(1-\gamma)} \int_{0}^{t_{2}}\left(t_{2}-r\right)^{\alpha-1} T_{\alpha}\left(t_{2}-r\right) g(r, u(r),(\mathcal{B} u) r) d r\right\| \rightarrow 0, \quad \text { as } t_{2} \rightarrow 0 . \\
\quad & \text { Now, let } 0<t_{1}<t_{2} \leq T . \text { One has } \\
\left\|\mathcal{P} y\left(t_{2}\right)-\mathcal{P} y\left(t_{1}\right)\right\| \leq & \left\|t_{2}^{(1+\alpha \beta)(1-\gamma)} S_{\alpha, \gamma}\left(t_{2}\right)\left[u_{0}-h(u(t))\right]-t_{1}^{(1+\alpha \beta) 1-\gamma} S_{\alpha, \gamma}\left(t_{1}\right)\left[u_{0}-h(u(t))\right]\right\| \\
& \quad+\| t_{2}^{(1+\alpha \beta)(1-\gamma)} \int_{0}^{t_{2}}\left(t_{2}-r\right)^{\alpha-1} T_{\alpha}\left(t_{2}-r\right) g(r, u(r),(\mathcal{B} u) r) d r \\
& \quad-t_{1}^{(1+\alpha \beta)(1-\gamma)} \int_{0}^{t_{1}}\left(t_{1}-r\right)^{\alpha-1} T_{\alpha}\left(t_{1}-r\right) g(r, u(r),(\mathcal{B} u) r) d r \| .
\end{aligned}
$$

Using the triangle inequality, we get

$$
\begin{aligned}
\left\|\mathcal{P} y\left(t_{2}\right)-\mathcal{P} y\left(t_{1}\right)\right\| \leq & \left\|t_{2}^{(1+\alpha \beta)(1-\gamma)} S_{\alpha, \gamma}\left(t_{2}\right)\left[u_{0}-h(u(t))\right]-t_{1}^{(1+\alpha \beta)(1-\gamma)} S_{\alpha, \gamma}\left(t_{1}\right)\left[u_{0}-h(u(t))\right]\right\| \\
& +\left\|t_{2}^{(1+\alpha \beta)(1-\gamma)} \int_{t_{1}}^{t_{2}}\left(t_{2}-r\right)^{\alpha-1} T_{\alpha}\left(t_{2}-r\right) g(r, u(r),(\mathcal{B} u) r) d r\right\| \\
& +\| t_{2}^{(1+\alpha \beta)(1-\gamma)} \int_{0}^{t_{1}}\left(t_{2}-r\right)^{\alpha-1} T_{\alpha}\left(t_{2}-r\right) g(r, u(r),(B u) r) d r \\
& -t_{1}^{(1+\alpha \beta)(1-\gamma)} \int_{0}^{t_{1}}\left(t_{1}-r\right)^{\alpha-1} T_{\alpha}\left(t_{2}-r\right) g(r, u(r),(\mathcal{B} u) r) d r \|
\end{aligned}
$$




$$
\begin{aligned}
& +\| t_{1}^{(1+\alpha \beta)(1-\gamma)} \int_{0}^{t_{1}}\left(t_{1}-r\right)^{\alpha-1} T_{\alpha}\left(t_{2}-r\right) g(r, u(r),(\mathcal{B} u) r) d r \\
& -t_{1}^{(1+\alpha \beta)(1-\gamma)} \int_{0}^{t_{1}}\left(t_{1}-r\right)^{\alpha-1} T_{\alpha}\left(t_{1}-r\right) g(r, u(r),(\mathcal{B} u) r) d r \| \\
= & : \mathfrak{I}_{1}+\mathfrak{I}_{2}+\mathfrak{I}_{3}+\mathfrak{I}_{4} .
\end{aligned}
$$

From the strong continuity of $S_{\alpha, \gamma}(t)$, we have $\mathfrak{I}_{1} \rightarrow 0$ as $t_{2} \rightarrow t_{1}$. Also,

$$
\begin{aligned}
\mathfrak{I}_{2} \leq & C_{2} t_{2}^{(1+\alpha \beta)(1-\gamma)} \int_{t_{1}}^{t_{2}}\left(t_{2}-r\right)^{-\alpha \beta-1}\left[k_{1}(r)+k_{2}(r) e^{-\delta r}\right] d r \\
\leq & C_{2} \mid t_{2}^{(1+\alpha \beta)(1-\gamma)} \int_{0}^{t_{2}}\left(t_{2}-r\right)^{-\alpha \beta-1}\left[k_{1}(r)+k_{2}(r) e^{-\delta r}\right] d r \\
& \quad-t_{2}^{(1+\alpha \beta)(1-\gamma)} \int_{0}^{t_{1}}\left(t_{1}-r\right)^{-\alpha \beta-1}\left[k_{1}(r)+k_{2}(r) e^{-\delta r}\right] d r \mid \\
& \leq C_{2} \int_{0}^{t_{1}}\left|t_{1}^{(1+\alpha \beta)(1-\gamma)}\left(t_{1}-r\right)^{-\alpha \beta-1}-t_{2}^{(1+\alpha \beta)(1-\gamma)}\left(t_{2}-r\right)^{-\alpha \beta-1}\right|\left[k_{1}(r)+k_{2}(r) e^{-\delta r}\right] d r .
\end{aligned}
$$

Since

Then, by using (H2) and the dominated convergence theorem, $\Im_{2} \rightarrow 0$ as $t_{2} \rightarrow t_{1}$.

$$
\begin{aligned}
\mathfrak{I}_{3} \leq C_{2} \int_{0}^{t_{1}}\left(t_{2}-r\right)^{-\alpha-\alpha \beta}\left|t_{2}^{(1+\alpha \beta)(1-\gamma)}\left(t_{2}-r\right)^{\alpha-1}-t_{1}^{(1+\alpha \beta)(1-\gamma)}\left(t_{1}-r\right)^{\alpha-1}\right|\left[k_{1}(r)+k_{2}(r) e^{-\delta r}\right] d r, \\
\left(t_{2}-r\right)^{-\alpha-\alpha \beta}\left|t_{2}^{(1+\alpha \beta)(1-\gamma)}\left(t_{2}-r\right)^{\alpha-1}-t_{1}^{(1+\alpha \beta)(1-\gamma)}\left(t_{1}-r\right)^{\alpha-1}\right|\left[k_{1}(r)+k_{2}(r) e^{-\delta r}\right] \\
\quad \leq t_{2}^{(1+\alpha \beta)(1-\gamma)}\left(t_{2}-r\right)^{\alpha-1}\left[k_{1}(r)+k_{2}(r) e^{-\delta r}\right]+t_{1}^{(1+\alpha \beta)(1-\gamma)}\left(t_{1}-r\right)^{\alpha-1}\left[k_{1}(r)+k_{2}(r) e^{-\delta r}\right] \\
\quad \leq 2 t_{1}^{(1+\alpha \beta)(1-\gamma)}\left(t_{1}-r\right)^{\alpha-1}\left[k_{1}(r)+k_{2}(r) e^{-\delta r}\right]
\end{aligned}
$$

and $\int_{0}^{t_{1}} 2 t_{1}^{(1+\alpha \beta)(1-\gamma)}\left(t_{1}-r\right)^{\alpha-1}\left[k_{1}(r)+k_{2}(r) e^{-\delta r}\right] d r$ exists, we obtain $I_{3} \rightarrow 0$ as $t_{2} \rightarrow t_{1}$. For $\epsilon>0$, we have

$$
\begin{aligned}
\mathfrak{I}_{4}= & \left\|\int_{0}^{t_{1}} t_{1}^{(1+\alpha \beta)(1-\gamma)}\left[T_{\alpha}\left(t_{2}-r\right)-T_{\alpha}\left(t_{1}-r\right)\right]\left(t_{1}-r\right)^{\alpha-1} g(r, u(r),(\mathcal{B} u) r) d r\right\| \\
\leq & \int_{0}^{t_{1}-\epsilon} t_{1}^{(1+\alpha \beta)(1-\gamma)}\left\|T_{\alpha}\left(t_{2}-r\right)-T_{\alpha}\left(t_{1}-r\right)\right\|_{L(\mathcal{Y})}\left(t_{1}-r\right)^{\alpha-1}\left[k_{1}(r)+k_{2}(r) e^{-\delta r}\right] \\
& +\int_{t_{1}-\epsilon}^{t_{1}} t_{1}^{(1+\alpha \beta)(1-\gamma)}\left\|T_{\alpha}\left(t_{2}-r\right)-T_{\alpha}\left(t_{1}-r\right)\right\|_{L(\mathcal{Y})}\left(t_{1}-r\right)^{\alpha-1}\left[k_{1}(r)+k_{2}(r) e^{-\delta r}\right] \\
\leq & t_{1}^{(1+\alpha \beta)(1-\gamma)} \int_{0}^{t_{1}}\left(t_{1}-r\right)^{\alpha-1}\left[k_{1}(r)+k_{2}(r) e^{-\delta r}\right] d r \sup _{s \in\left[0, t_{1}-\epsilon\right]}\left\|T_{\alpha}\left(t_{2}-r\right)-T_{\alpha}\left(t_{1}-r\right)\right\|_{L(\mathcal{Y})} \\
& +C_{2} \int_{t_{1}-\epsilon}^{t_{1}} t_{1}^{(1+\alpha \beta)(1-\gamma)}\left(\left(t_{2}-r\right)^{-\alpha-\alpha \beta}+\left(t_{1}-r\right)^{-\alpha-\alpha \beta}\right)\left(t_{1}-r\right)^{\alpha-1}\left[k_{1}(r)+k_{2}(r) e^{-\delta r}\right] d r \\
\leq & t_{1}^{(1+\alpha \beta)(1-\gamma)+\alpha(1+\beta)} \int_{0}^{t_{1}}\left(t_{1}-r\right)^{-\alpha \beta-1}\left[k_{1}(r)+k_{2}(r) e^{-\delta r}\right] d r \sup _{s \in\left[0, t_{1}-\epsilon\right]}\left\|T_{\alpha}\left(t_{2}-r\right)-T_{\alpha}\left(t_{1}-r\right)\right\|_{L(\mathcal{Y})} \\
& +2 C_{2} \int_{t_{1}-\epsilon}^{t_{1}} t_{1}^{(1+\alpha \beta)(1-\gamma)}\left(t_{1}-r\right)^{-\alpha \beta-1}\left[k_{1}(r)+k_{2}(r) e^{-\delta r}\right] d r .
\end{aligned}
$$

Since $T_{\alpha}(t)$ is uniformly continuous and $\lim _{t_{2} \rightarrow t_{1}} \mathfrak{I}_{2}=0$, then $\mathfrak{I}_{4} \rightarrow 0$ as $t_{2} \rightarrow t_{1}$, independent of $y \in \Omega_{r}(\mathcal{J})$. Hence, $\left\|\mathcal{P} y\left(t_{2}\right)-\mathcal{P} y\left(t_{1}\right)\right\| \rightarrow 0$, independently of $y \in \Omega_{r}(\mathcal{J})$ as $t_{2} \rightarrow t_{1}$. Therefore, $\left\{\mathcal{P} y: y \in \Omega_{r}(\mathcal{J})\right\}$ is equicontinuous. 
Theorem 4. Let $-1<\beta<0,0<\omega<\frac{\pi}{2}$, and $\mathcal{A} \in \Theta_{\omega}^{\beta}$. Then, under hypotheses (H1)-(H4), the operator $\left\{\mathcal{P} y: y \in \Omega_{r}(\mathcal{J})\right\}$ is continuous and bounded, provided $u_{0} \in \mathcal{D}\left(\mathcal{A}^{\theta}\right)$ with $\theta>1+\beta$.

Proof. We verify that $\mathcal{P}$ maps $\Omega_{r}(\mathcal{J})$ into itself. Taking $y \in \Omega_{r}(\mathcal{J})$ and defining

$$
u(t):=t^{-(1+\alpha \beta)(1-\gamma)} y(t),
$$

we have $u \in \Omega_{r}(\mathcal{J})$. Let $t \in[0, T]$,

$$
\begin{aligned}
& \|\mathcal{P}\| \leq\left\|t^{(1+\alpha \beta)(1-\gamma)} S_{\alpha, \gamma}(t)\left[u_{0}-h(u(t))\right]\right\|+t^{(1+\alpha \beta)(1-\gamma)}\left\|\int_{0}^{t}(t-r)^{\alpha-1} T_{\alpha}(t-r) g(r, u(r),(\mathcal{B} u) r) d r\right\| . \\
& \qquad \begin{aligned}
\| r o m(\mathrm{H} 2)-(\mathrm{H} 4), \text { we get } \\
\|\mathcal{P} y(t)\| \leq t^{(1+\alpha \beta)(1-\gamma)}\left\|S_{\alpha, \gamma}(t)\left[u_{0}-h(u(t))\right]\right\|+t^{(1+\alpha \beta)(1-\gamma)} \int_{0}^{t}(t-r)^{-\alpha \beta-1}\left[k_{1}(r)+k_{2}(r) e^{-\delta r}\right] d r \\
\leq \sup _{[0, T]}\left(t^{(1+\alpha \beta)(1-\gamma)}\left\|S_{\alpha, \gamma}(t)\right\|\left[\left\|u_{0}\right\|+k\right]+t^{(1+\alpha \beta)(1-\gamma)} \int_{0}^{t}(t-r)^{-\alpha \beta-1}\left[k_{1}(r)+k_{2}(r) e^{-\delta r}\right] d r\right) \\
\leq r .
\end{aligned}
\end{aligned}
$$

Thus, $\|\mathcal{P} y\| \leq r$ for any $y \in \Omega_{r}(\mathcal{J})$. Now, to verify $\mathcal{P}$ is continuous in $\Omega_{r}(\mathcal{J})$, let $y_{n}, y \in \Omega_{r}(\mathcal{J}), n=1,2, \ldots$ with $\lim _{n \rightarrow \infty} y_{n}=y$; that is, $\lim _{n \rightarrow \infty} y_{n}(t)=y(t)$; $\lim _{n \rightarrow \infty} t^{-(1+\alpha \beta) 1-\gamma} \quad y_{n}(t) \quad=\quad t^{-(1+\alpha \beta) 1-\gamma} \quad y(t) \quad$ and $\lim _{n \rightarrow \infty} t^{-(1+\alpha \beta)(1-\gamma)} y_{n}(t)=t^{-(1+\alpha \beta)(1-\gamma)} y(t)$ on $\mathcal{J}$. Then, (H1) implies that

$$
\begin{aligned}
g\left(t, u_{n}(t), \mathcal{B}\left(u_{n}(t)\right)\right)= & g\left(t, t^{-(1+\alpha \beta)(1-\gamma)} y_{n}(t), t^{-(1+\alpha \beta)(1-\gamma)} \mathcal{B}\left(y_{n}(t)\right)\right) \\
& \rightarrow g\left(t, t^{-(1+\alpha \beta)(1-\gamma)} y(t), t^{-(1+\alpha \beta)(1-\gamma)} \mathcal{B}(y(t))\right)
\end{aligned}
$$

as $n \rightarrow \infty$. From (H2), we obtain the inequality

$$
(t-r)^{-\alpha \beta-1}\left|g\left(r, u_{n}(r), \mathcal{B}\left(u_{n}(r)\right)\right)\right| \leq 2(t-r)^{-(\alpha \beta)(1-\gamma)}\left[k_{1}(r)+k_{2}(r) e^{-\delta r}\right],
$$

that is,

$$
\int_{0}^{t}(t-r)^{-\alpha \beta-1} \| g\left(r, u_{n}(r), \mathcal{B}\left(u_{n}(r)\right)\right)-g(r, u(r), \mathcal{B}(u(r)) \| d r \rightarrow 0 \quad \text { as } n \rightarrow \infty .
$$

Let $t \in[0, T]$. Now,

$$
\left\|\mathcal{P} y_{n}(t)-\mathcal{P} y(t)\right\| \leq t^{(1+\alpha \beta)(1-\gamma)} \| \int_{0}^{t}(t-r)^{\alpha-1} T_{\alpha}(t-r)\left(g \left(r, u_{n}(r), \mathcal{B}\left(u_{n}(r)\right)-g(r, u(r), \mathcal{B}(u(r))) d r \| .\right.\right.
$$

Applying Theorem 1, we have

$$
\left\|\mathcal{P} y_{n}(t)-\mathcal{P} y(t)\right\| \leq C_{2} t^{(1+\alpha \beta)(1-\gamma)} \int_{0}^{t}(t-r)^{-\alpha \beta-1} \| g\left(r, u_{n}(r), \mathcal{B}\left(u_{n}(r)\right)-g(r, u(r), \mathcal{B}(u(r)) \| d r,\right.
$$

which tends to 0 as $n \rightarrow \infty$, i.e., $\mathcal{P} y_{n} \rightarrow \mathcal{P} y$ pointwise on $\mathcal{J}$. Moreover, Theorem 3 implies that $\mathcal{P} y_{n} \rightarrow \mathcal{P} y$ uniformly on $\mathcal{J}$ as $n \rightarrow \infty$, that is, $\mathcal{P}$ is continuous.

\section{Main Results}

We prove the existence of a mild solution to the problem in Equations (1) and (2) when the associated semigroup is compact (Theorem 5) and noncompact (Theorem 6).

\subsection{Compactness of the Semigroup}

Here, we assume $Q(t)$ to be compact. 
Theorem 5. Let $-1<\beta<0,0<\omega<\frac{\pi}{2}$ and $\mathcal{A} \in \Theta_{\omega}^{\beta}$. If $Q(t)(t>0)$ is compact and (H1)-(H4) hold, then there exists a mild solution of (1)-(2) in $\Omega_{r}(\mathcal{J})$ for every $u_{0} \in D\left(\mathcal{A}^{\theta}\right)$ with $\theta>1+\beta$.

Proof. Because we assume $Q(t)$ to be compact, then the equicontinuity of $Q(t)(t>0)$ is ensured. Moreover, by Theorems 3 and $4, \mathcal{P}: \Omega_{r}(\mathcal{J}) \rightarrow \Omega_{r}(\mathcal{J})$ is continuous and bounded and $\varepsilon: \Omega_{r}(\mathcal{J}) \rightarrow \Omega_{r}(\mathcal{J})$ is bounded and continuous, and $\left\{\varepsilon y: y \in \Omega_{r}(\mathcal{J})\right\}$ is equicontinuous. We can write $\epsilon: \Omega_{r}(\mathcal{J}) \rightarrow \Omega_{r}(\mathcal{J})$ by $(\epsilon y)(t)=\left(\epsilon^{1} y\right)(t)+\left(\epsilon^{2} y\right)(t)$, where

$$
\begin{aligned}
\left(\epsilon^{1} y\right)(t) & =t^{(1+\alpha \beta)(1-\gamma)} S_{\alpha, \gamma}(t)\left(u_{0}-h(u)\right)=t^{(1+\alpha \beta)(1-\gamma)} \mathcal{I}_{0^{+}}^{\gamma(1-\alpha)} t^{\alpha-1} T_{\alpha}(t)\left(u_{0}-h(u)\right) \\
& =\frac{t^{(1+\alpha \beta)(1-\gamma)}}{\Gamma(\gamma(1-\alpha))} \int_{0}^{t}(t-r)^{\gamma(1-\alpha)-1} r^{\alpha-1} \int_{0}^{\infty} \alpha \theta M_{\alpha}(\theta) Q\left(r^{\alpha} \theta\right)\left(u_{0}-h(u)\right) d \theta d r \\
& =\frac{\alpha t^{(1+\alpha \beta)(1-\gamma)}}{\Gamma(\gamma(1-\alpha))} \int_{0}^{t} \int_{0}^{\infty}(t-r)^{\gamma(1-\alpha)-1} r^{\alpha-1} \theta M_{\alpha}(\theta) Q\left(r^{\alpha} \theta\right)\left(u_{0}-h(u)\right) d \theta d r
\end{aligned}
$$

and

$$
\left(\varepsilon^{2} y\right)(t)=t^{(1+\alpha \beta)(1-\gamma)} \int_{0}^{t}(t-r)^{\alpha-1} T_{\alpha}(t-r) g(r, u(r)(\mathcal{B} u) r) d r .
$$

For $\sigma>0$ and $\theta \in(0, t)$, we define an operator $\varepsilon_{\theta, \sigma}^{1}$ on $\Omega_{r}(\mathcal{J})$ by

$$
\begin{aligned}
\left(\varepsilon_{\theta, \sigma}^{1} y\right)(t) & =\frac{t^{(1+\alpha \beta)(1-\gamma)}}{\Gamma(\gamma(1-\alpha))} \int_{\theta}^{t} \int_{\sigma}^{\infty}(t-r)^{(1-\alpha) \gamma-1} r^{\alpha-1} \theta M_{\alpha}(\theta) Q\left(r^{\alpha} \theta\right)\left(u_{0}-h(u)\right) d \theta d r \\
& =\frac{\alpha t^{(1+\alpha \beta)(1-\gamma)}}{\Gamma(\gamma(1-\alpha))} \mathfrak{T}\left(\theta^{\alpha} \sigma\right) \int_{\theta}^{t} \int_{\sigma}^{\infty}(t-r)^{(1-\alpha) \gamma-1} r^{\alpha-1} \theta M_{\alpha}(\theta) Q\left(r^{\alpha} \theta-\theta^{\alpha} \sigma\right)\left(u_{0}-h(u)\right) d \theta d r .
\end{aligned}
$$

Since $\mathfrak{T}\left(\epsilon^{\alpha} \delta\right)$ is compact, $\left.\mathcal{V}_{\theta, \sigma}^{1}(t)=\left\{\varepsilon_{\theta, \sigma}^{1} y\right)(t), y \in \Omega_{r}(\mathcal{J})\right\}$ is precompact in $\mathcal{Y}$ for all $\theta \in(0, t)$ and $\delta>0$. Moreover, for any $y \in \Omega_{r}(\mathcal{J})$, one has

$$
\begin{aligned}
&\left\|\left(\varepsilon^{1} y\right)(t)-\left(\varepsilon_{\theta, \sigma}^{1} y\right)(t)\right\| \\
& \leq \mathcal{K}(\alpha, \gamma)\left\|t^{(1+\alpha \beta)(1-\gamma)} \int_{0}^{t} \int_{0}^{\sigma}(t-r)^{\gamma(1-\alpha)-1} r^{\alpha-1} \theta M_{\alpha}(\theta) Q\left(r^{\alpha} \theta\right)\left(u_{0}-h(u)\right) d \theta d r\right\| \\
&+\mathcal{K}(\alpha, \gamma)\left\|t^{(1+\alpha \beta)(1-\gamma)} \int_{0}^{\theta} \int_{\sigma}^{\infty}(t-r)^{\gamma(1-\alpha)-1} r^{\alpha-1} \theta M_{\alpha}(\theta) Q\left(r^{\alpha} \theta\right)\left(u_{0}-h(u)\right) d \theta d r\right\| \\
& \leq \mathcal{K}(\alpha, \gamma) t^{(1+\alpha \beta)(1-\gamma)} \int_{0}^{t} \int_{0}^{\sigma}(t-r)^{\gamma(1-\alpha)-1} r^{\alpha-1} \theta M_{\alpha}(\theta) r^{-\alpha \gamma-\alpha}\left\|\left(u_{0}-h(u)\right)\right\| \theta^{-\beta-1} d \theta d r \\
&+\mathcal{K}(\alpha, \gamma) t^{(1+\alpha \beta)(1-\gamma)} \int_{0}^{\theta} \int_{\sigma}^{\infty}(t-r)^{\gamma(1-\alpha)-1} r^{\alpha-1} \theta M_{\alpha}(\theta) r^{-\alpha \beta-\alpha} \theta^{-\beta-1}\left\|\left(u_{0}-h(u)\right)\right\| d \theta d r \\
&= \mathcal{K}(\alpha, \gamma) t^{(1+\alpha \beta)(1-\gamma)} \int_{0}^{t}(t-r)^{\gamma(1-\alpha)-1} r^{-\alpha \beta-1}\left\|\left(u_{0}-h(u)\right)\right\| d r \int_{0}^{\sigma} \theta^{-\beta} M_{\alpha}(\theta) d \theta \\
&+\mathcal{K}(\alpha, \gamma) t^{(1+\alpha \beta)(1-\gamma)} \int_{0}^{\theta}(t-r)^{\gamma(1-\alpha)-1} r^{-\alpha \beta-1}\left\|\left(u_{0}-h(u)\right)\right\| d r \int_{\eta}^{\infty} \theta^{-\beta} M_{\alpha}(\theta) d \theta \\
& \leq \mathcal{K} t^{-\alpha \gamma(1+\beta)}\left\|\left(u_{0}-h(u)\right)\right\| \int_{0}^{\eta} \theta^{-\beta} M_{\alpha}(\theta) d \theta \\
&+\mathcal{K} t^{-\alpha \gamma(1+\beta)}\left[\left\|u_{0}\right\|+k\right] \int_{0}^{\theta}(1-s)^{\gamma(1-\alpha)-1} r^{-\alpha \beta-1} d r \int_{\eta}^{\infty} \theta^{-\beta} M_{\alpha}(\theta) d \theta \\
& \rightarrow 0 \text { as } \theta \rightarrow 0 \text { and } \sigma \rightarrow 0,
\end{aligned}
$$

where $\mathcal{K}(\alpha, \gamma)=\frac{\alpha}{\Gamma(\gamma(1-\alpha))}$. Therefore, $\mathcal{V}_{\theta, \sigma}^{1}(t)=\left\{\left(\varepsilon_{\theta, \sigma}^{1} y\right)(t), y \in \Omega_{r}(\mathcal{J})\right\}$ are arbitrarily close to $\mathcal{V}^{1}(t)=\left\{\left(\varepsilon^{1} y\right)(t), y \in \Omega_{r}(\mathcal{J})\right\}$ for $t>0$. Hence, $\mathcal{V}^{1}(t)$, for $t>0$, is precompact in $\mathcal{Y}$. For $\theta \in(0, t)$ and $\sigma>0$, we can present an operator $\varepsilon_{\theta, \sigma}^{2}$ on $\Omega_{r}(\mathcal{J})$ by 


$$
\begin{aligned}
\left(\varepsilon_{\theta, \sigma}^{2} y\right)(t) & =\alpha t^{(1+\alpha \beta)(1-\gamma)} \int_{0}^{t-\theta} \int_{\sigma}^{\infty} \theta M_{\alpha}(\theta)(t-r)^{\alpha-1} Q\left((t-r)^{\alpha} \theta\right) g(r, u(r),(\mathcal{B} u) r) d \theta d r \\
& =\alpha t^{(1+\alpha \beta)(1-\gamma)} \mathfrak{T}\left(\theta^{\alpha} \sigma\right) \int_{0}^{t-\theta} \int_{\sigma}^{\infty} \theta M_{\alpha}(\theta)(t-r)^{\alpha-1} Q\left((t-r)^{\alpha} \theta-\theta^{\alpha} \sigma\right) g(r, u(r),(\mathcal{B} u) r) d \theta d r .
\end{aligned}
$$

Thus, due to the compactness of $Q\left(\theta^{\alpha} \sigma\right), \mathcal{V}_{\theta, \sigma}^{2}(t)=\left\{\left(\varepsilon_{\theta, \sigma}^{2} y\right)(t), y \in \Omega_{r}(\mathcal{J})\right\}$ is precompact in $\mathcal{Y}$ for all $\theta \in(0, t)$ and $\sigma>0$. For every $y \in \Omega_{r}(\mathcal{J})$, we get

$$
\begin{aligned}
& \left\|\left(\varepsilon^{2} y\right)(t)-\left(\varepsilon_{\theta, \sigma}^{2} y\right)(t)\right\| \\
& \leq\left\|\alpha t^{(1+\alpha \beta)(1-\gamma)}\left(\int_{0}^{t} \int_{0}^{\sigma} \theta M \alpha(\theta)(t-r)^{\alpha-1} Q\left((t-r)^{\alpha} \theta\right) g(r, u(r),(\mathcal{B} u) r) d \theta d r\right)\right\| \\
& +\left\|\alpha t^{(1+\alpha \beta)(1-\gamma)}\left(\int_{t-\theta}^{t} \int_{\sigma}^{\infty}(t-r)^{\alpha-1} \theta M_{\alpha}(\theta) Q\left((t-r)^{\alpha} \theta\right) g(r, u(r),(\mathcal{B} u) r) d \theta d r\right)\right\| \\
& \leq \alpha C_{0} t^{(1+\alpha \beta)(1-\gamma)}\left(\int_{0}^{t}(t-r)^{-\alpha \beta-1}\left[k_{1}(r)+k_{2}(r) e^{-\delta r}\right] d r \int_{0}^{\sigma} \theta^{-\beta} M_{\alpha}(\theta) d \theta\right) \\
& \quad+\alpha C_{0} t^{(1+\alpha \beta)(1-\gamma)}\left(\int_{t-\theta}^{t}(t-r)^{-\alpha \beta-1}\left[k_{1}(r)+k_{2}(r) e^{-\delta r}\right] d r \int_{0}^{\infty} \theta^{-\beta} M_{\alpha}(\theta) d \theta\right) \\
& \leq \alpha C_{0} t^{(1+\alpha \beta)(1-\gamma)}\left(\int_{0}^{t}(t-r)^{-\alpha \beta-1}\left[k_{1}(r)+k_{2}(r) e^{-\delta r}\right] d r \int_{0}^{\sigma} \theta^{-\beta} M_{\alpha}(\theta) d \theta\right) \\
& \quad+\frac{\alpha C_{0} \Gamma(1-\beta)}{\Gamma(1-\alpha \beta)} t^{(1+\alpha \beta)(1-\gamma)}\left(\int_{t-\theta}^{t}(t-r)^{-\alpha \beta-1}\left[k_{1}(r)+k_{2}(r) e^{-\delta r}\right] d r\right) \rightarrow 0 \text { as } \sigma \rightarrow 0 .
\end{aligned}
$$

Therefore, $\mathcal{V}_{\theta, \sigma}^{2}(t)=\left\{\left(\varepsilon_{\theta, \sigma}^{2} y\right)(t), y \in \Omega_{r}(\mathcal{J})\right\}$ are arbitrarily close to $\mathcal{V}^{2}(t)=\left\{\left(\varepsilon^{2} y\right)(t)\right.$, $\left.y \in \Omega_{r}(\mathcal{J})\right\}, t>0$. This implies the relative compactness of $\mathcal{V}^{2}(t), t>0$, in $\mathcal{Y}$. Also, $\mathcal{V}(t)=\left\{(\varepsilon y)(t), y \in \Omega_{r}(\mathcal{J})\right\}$ is relatively compact in $\mathcal{Y} \forall t \in[0, T]$. It follows, from the Arzela-Ascoli theorem, that $\left\{\varepsilon y, y \in \Omega_{r}(\mathcal{J})\right\}$ is relatively compact for $\varepsilon$, it is continuous, and $\left\{\varepsilon y, y \in \Omega_{r}(\mathcal{J})\right\}$ is relatively compact. This implies, by the Schauder fixed point theorem, the existence of a fixed point $y^{*} \in \Omega_{r}(\mathcal{J})$ of $\varepsilon$. Let $u^{*}(t):=t^{(1+\alpha \beta)(\gamma-1)} y^{*}(t)$. Then, $u^{*}$ is a mild solution of Equations (1) and (2).

\subsection{Non-Compactness of the Semigroup}

Now, we assume that $Q(t)$ is noncompact. We need the following supplementary condition:

Hypothesis 5 (H5). There exists a constant $k>0$ satisfying

$$
\Theta\left(g\left(t, \mathfrak{E}_{1}, \mathfrak{E}_{2}\right)\right) \leq k \Theta\left(\mathfrak{E}_{1}, \mathfrak{E}_{2}\right) \text { for almost all } t \in[0, T]
$$

and for every bounded subsets $\mathfrak{E}_{1}, \mathfrak{E}_{2} \subset \mathcal{Y}$.

Theorem 6. Let $-1<\beta<0,0<\omega<\frac{\pi}{2}$, and $\mathcal{A} \in \Theta_{\omega}^{\beta}$. Suppose (H1)-(H5) hold. Then, problem (1)-(2) has a mild solution in $\Omega_{r}(\mathcal{J})$ for every $u_{0} \in D\left(g^{\theta}\right)$ with $\theta>1+\beta$.

Proof. From Theorems 3 and 4, we find that $\varepsilon: \Omega_{r}(\mathcal{J}) \rightarrow \Omega_{r}(\mathcal{J})$ is continuous and bounded, and $\left\{\varepsilon y: y \in \Omega_{r}(\mathcal{J})\right\}$ is equicontinuous. Furthermore, we prove that there is a subset of $\Omega_{r}(\mathcal{J})$ such that $\varepsilon$ is compact in it. For any bounded set $\mathbb{P}_{0} \subset \Omega_{r}(\mathcal{J})$, set

$$
\varepsilon^{(1)}\left(\mathbb{P}_{0}\right)=\varepsilon\left(\mathbb{P}_{0}\right), \varepsilon^{(n)}\left(\mathbb{P}_{0}\right)=\varepsilon\left(\overline{c o}\left(\varepsilon^{(n-1)}\left(\mathbb{P}_{0}\right)\right)\right), \quad n=2,3, \ldots
$$

For any $\epsilon>0$, we can obtain from Propositions 1-3 a subsequence $\left\{y_{n}^{(1)}\right\}_{n=1}^{\infty} \subset \mathbb{P}_{0}$ satisfying 


$$
\begin{aligned}
\Theta\left(\varepsilon^{(1)}\left(\mathbb{P}_{0}(t)\right)\right) & \leq 2 \Theta\left(t^{(1+\alpha \beta)(1-\gamma)} \int_{0}^{t}(t-r)^{\alpha-1} T_{\alpha}(t-r) g\left(r,\left\{r^{-(1+\alpha \beta)(1-\gamma)}\left(y_{n}^{(1)}(r), \mathcal{B} y_{n}^{(1)}(r)\right)\right\}_{n=1}^{\infty}\right) d r\right) \\
& \leq 4 C_{p} t^{(1+\alpha \beta)(1-\gamma)}\left(\int_{0}^{t}(t-r)^{-\alpha \beta-1} \Theta\left(g\left(r,\left\{r^{-(1+\alpha \beta)(1-\gamma)}\left(y_{n}^{(1)}(r), \mathcal{B} y_{n}^{(1)}(r)\right)\right\}_{n=1}^{\infty}\right)\right) d r\right) \\
& \leq 4 C_{p} k t^{(1+\alpha \beta)(1-\gamma)} \Theta\left(\mathfrak{P}_{0}\right)\left(\int_{0}^{t}(t-r)^{-\alpha \beta-1} r^{-(1+\alpha \beta)(1-\gamma)} d r\right) \\
& =4 C_{p} k t^{-\alpha \beta} \Theta\left(\mathfrak{P}_{0}\right)\left(\frac{\Gamma(-\alpha \beta) \Gamma((-\alpha \beta+\gamma(1+\alpha \beta))}{\Gamma(-2 \alpha \beta+\gamma(1+\alpha \beta))}\right) .
\end{aligned}
$$

Since $\epsilon$ is arbitrary, then

$$
\Theta\left(\varepsilon^{(1)}\left(\mathbb{P}_{0}(t)\right)\right) \leq 4 C_{p} k t^{-\alpha \beta} \Theta\left(\mathbb{P}_{0}\right)\left(\frac{\Gamma(-\alpha \beta) \Gamma((-\alpha \beta+\gamma(1+\alpha \beta))}{\Gamma(-2 \alpha \beta+\gamma(1+\alpha \beta))}\right) .
$$

Again, for any $\epsilon>0$, we can get from Propositions 1-3 a subsequence $\left\{y_{n}^{(2)}, \mathcal{B} y_{n}^{(2)}\right\}_{n=1}^{\infty} \subset$ $\overline{c o}\left(\varepsilon^{(1)}\left(\mathbb{P}_{0}\right)\right)$, which implies that

$$
\begin{aligned}
\Theta\left(\varepsilon^{(2)}\left(\mathbb{P}_{0}(t)\right)\right)= & \Theta\left(\varepsilon\left(\overline{c o}\left(\varepsilon^{(1)}\left(\mathbb{P}_{0}(t)\right)\right)\right)\right) \\
\leq & 2 \Theta\left(t^{(1+\alpha \beta)(1-\gamma)} \int_{0}^{t}(t-r)^{\alpha-1} \mathcal{Q}_{\alpha}(t-r) g\left(r,\left\{r^{-(1+\alpha \beta)(1-\gamma)}\left(y_{n}^{(2)}(r), \mathcal{B} y_{n}^{(2)}(r)\right)\right\}_{n=1}^{\infty}\right) d r\right) \\
\leq & 4 C_{p} t^{(1+\alpha \beta)(1-\gamma)}\left(\int_{0}^{t}(t-r)^{-\alpha \beta-1} \Theta\left(g\left(r,\left\{r^{-(1+\alpha \beta)(1-\gamma)}\left(y_{n}^{(2)}(r), \mathcal{B} y_{n}^{(2)}(r)\right)\right\}_{n=1}^{\infty}\right) d r\right)\right. \\
\leq & 4 C_{p} k t^{(1+\alpha \beta)(1-\gamma)}\left(\int_{0}^{t}(t-r)^{-\alpha \beta-1} \Theta\left(r^{-(1+\alpha \beta)(1-\gamma)}\left(\left\{y_{n}^{(2)}(r), \mathcal{B} y_{n}^{(2)}(r)\right\}_{n=1}^{\infty}\right)\right) d r\right) \\
\leq & 4 C_{p} k t^{(1+\alpha \beta)(1-\gamma)}\left(\int_{0}^{t}(t-r)^{-\alpha \beta-1} r^{-(1+\alpha \beta)(1-\gamma)} \Theta\left(\left\{y_{n}^{(2)}(r), \mathcal{B} y_{n}^{(2)}\right\}_{n=1}^{\infty}\right) d r\right) \\
\leq & \frac{\left(4 C_{p} k\right)^{2} t^{(1+\alpha \beta)(1-\gamma)} \Gamma(-\alpha \beta) \Gamma(-\alpha \beta+\gamma(1+\alpha \beta))}{\Gamma(-2 \alpha \beta+\gamma(1+\alpha \beta))} \Theta\left(\mathbb{P}_{0}\right) \\
& \times\left(\int_{0}^{t}(t-r)^{-\alpha \beta-1} r^{-(1+\alpha \beta)(1-\gamma)-\alpha \beta} d r\right) \\
= & \left(\frac{\left(4 C_{p} k\right)^{2} t^{-2 \alpha \beta} \Gamma^{2}(-\alpha \beta) \Gamma(-\alpha \beta+\gamma(1+\alpha \beta))}{\Gamma(-3 \alpha \beta+\gamma(1+\alpha \beta))}\right) \Theta\left(\mathbb{P}_{0}\right) .
\end{aligned}
$$

Now,

$$
\Theta\left(\varepsilon^{(n)}\left(\mathbb{P}_{0}(t)\right)\right) \leq \frac{\left(4 C_{p} k\right)^{n} t^{-n \alpha \beta} \Gamma^{n}(-\alpha \beta) \Gamma(-\alpha \beta+\gamma(1+\alpha \beta))}{\Gamma(-(n+1) \alpha \beta+\gamma(1+\alpha \beta))} \Theta\left(\mathbb{P}_{0}\right), \quad n \in \mathbb{N} .
$$

Let $M=4 C_{p} k T^{-\alpha \beta} \Gamma(-\alpha \beta)$. We can find $m, k \in \mathbb{N}$ large enough such that $\frac{1}{k}<\alpha \beta<$ $\frac{1}{k-1}$ and $\frac{n+1}{k}>2$ for $n>m \Gamma(-(n+1) \alpha \beta+\gamma(1+\alpha \beta))>\Gamma\left(\frac{n+1}{k}\right)$, that is,

$$
\frac{\left(4 C_{p} k\right)^{n} T^{-n \alpha \beta} \Gamma^{n}(-\alpha \beta) \Gamma(-\alpha \beta+\gamma(1+\alpha \beta))}{\Gamma(-(n+1) \alpha \beta+\gamma(1+\alpha \beta))}<\frac{\left(4 C_{p} k\right)^{n} T^{-n \alpha \beta} \Gamma^{n}(-\alpha \beta) \Gamma(-\alpha \beta+\gamma(1+\alpha \beta))}{\Gamma\left(\frac{n+1}{k}\right)} .
$$
becomes

Replacing $(n+1)$ by $(j+1) k$, then the right-hand side of the inequality given above

$$
\frac{M^{(j+1) k-1} \Gamma(-\alpha \beta+\gamma(1+\alpha \beta))}{\Gamma(j+1)}=\frac{\left(M^{k}\right)^{j} M^{k-1} \Gamma(-\alpha \beta+\gamma(1+\alpha \beta))}{j !} \rightarrow 0 \text { as } j \rightarrow \infty .
$$

Therefore, there exists a constant $n_{0} \in \mathbb{N}$ such that 


$$
\frac{\left(4 C_{p} k^{n} t^{-n \alpha \beta} \Gamma^{n}(-\alpha \beta) \Gamma(-\alpha \beta+\gamma(1+\alpha \beta)\right.}{\Gamma(-(n+1) \alpha \beta+\gamma(1+\alpha \beta))} \leq \frac{\left(4 C_{p} k\right)^{n_{0}} T^{-n_{0} \alpha \beta} \Gamma^{n_{0}}(-\alpha \beta) \Gamma(-\alpha \beta+\gamma(1+\alpha \beta))}{\Gamma\left(-\left(n_{0}^{+} 1\right) \alpha \beta+\gamma(1+\alpha \beta)\right)}=p<1 .
$$

Now, $\Theta\left(\varepsilon^{\left(n_{0}\right)}\left(\mathbb{P}_{0}(t)\right)\right) \leq p \Theta\left(\mathbb{P}_{0}\right)$. Since $\varepsilon^{\left(n_{0}\right)}\left(\mathbb{P}_{0}(t)\right)$ is bounded and equicontinuous, it follows from Proposition 1 that

$$
\Theta\left(\varepsilon^{\left(n_{0}\right)}\left(\mathbb{P}_{0}\right)\right)=\max _{t \in[0, T]} \Theta\left(\varepsilon^{n_{0}}\left(\mathbb{P}_{0}(t)\right)\right) .
$$

Thus, $\Theta\left(\varepsilon^{n_{0}}\left(\mathbb{P}_{0}\right)\right) \leq p \Theta\left(\mathbb{P}_{0}\right)$, where $p<1$. Using a similar technique as in Theorem 5, we obtain $C$ in $\Omega_{r}(\mathcal{J})$ with $\varepsilon(C) \subset C$ and $\varepsilon(C)$ compact. By applying Schauder's fixed point theorem, we obtain a fixed point $y^{*}$ in $\Omega_{r}(\mathcal{J})$ of $\varepsilon$. Let $u^{*}(t)=t^{(1+\alpha \beta)(\gamma-1)} y^{*}(t)$. Then, $u^{*}(t)$ is a mild solution of problem (1)-(2).

\section{Example}

As an illustrative example, let us consider the following Hilfer fractional partial differential equation with a nonlocal condition:

$$
\begin{gathered}
D_{0^{+}}^{\frac{3}{4}, \frac{1}{2}} z(t, y)+\partial_{x}^{2} z(t, y)=G(t, z(t, y), \mathcal{B} z(t, y)), \quad t \in(0,1], \quad y \in[0, \pi], \\
z(t, 0)=z(t, \pi)=0, \quad t \in(0,1], \\
\left.I_{0^{+}}^{\frac{1}{8}}[z(t, y)]\right|_{t=0}+\sum_{i=1}^{m} c_{i} z\left(t_{i}, y\right)=z_{0}(y), \quad y \in[0, \pi]
\end{gathered}
$$

where $\alpha=\frac{3}{4}, \gamma=\frac{1}{2}, 0<t_{1}<\cdots<t_{m}<1$, and $c_{i}, i=1, \ldots, m$, are given constants. Let us take the nonlinear function $G(t, z(\cdot), \mathcal{B} z(\cdot))=y \cos z(t, y)+\int_{0}^{t} e^{-(t-s)} \sin z(t, y) d s$ and the nonlocal function $h(z(t, \cdot))=\sum_{i=1}^{m} c_{i} z\left(t_{k}, \cdot\right)$. Assume that $\mathcal{Y}=L^{2}[0, \pi]$ and define $\mathcal{A}: D(\mathcal{A}) \subset \mathcal{Y} \rightarrow \mathcal{Y}$ by $\mathcal{A} z=z_{y y}$ with domain

$$
D(\mathcal{A})=\left\{z \in \mathcal{Y}: z_{y}, z_{y y} \in \mathcal{Y}, z(t, 0)=z(t, \pi)=0\right\} .
$$

It follows from the work in [20] that there exist constants $\delta, \epsilon>0$ such that $\mathcal{A}+\delta \in$ $\odot \frac{\pi}{\frac{\pi}{2}-\epsilon}(\mathcal{Y})$. It is known that $\mathcal{A}$ is the infinitesimal generator of a differentiable semigroup $Q(t)(t>0)$ in $\mathcal{Y}$ given by

$$
(Q(t) x)(y)=\left\{\begin{array}{l}
\int_{0}^{\pi} \psi(t, y-s) x(s) d s, t>0, \\
x(y), \quad t=0
\end{array}\right.
$$

where

$$
\psi(t, y)=\frac{1}{\sqrt{4 \pi t}} e^{-\frac{y^{2}}{4 t}}, \quad t>0, \quad 0<y<\pi,
$$

and $x(t)(y)=z(t, y)$. This implies $\|Q(t)\| \leq 1$ and leads to its compactness property. We can check that all hypotheses (H1)-(H4) are fulfilled; thus, our Theorem 5 can be applied ensuring that problem (4) admits a mild solution.

\section{Conclusions}

In this paper, we applied Schauder's fixed point theorem to investigate the solvability of a class of Hilfer fractional integro-differential equations involving almost sectorial operators. We discussed cases of both compactness and noncompactness related to associated semigroup operators. The obtained existence results were subject to an appropriate set of sufficient conditions. As a future direction of research, it would be desirable to consider the study of $\psi$-Hilfer fractional nonlocal nonlinear stochastic systems involving almost sectorial operators and impulsive effects, generalizing the current work. Another open line 
of research consists of the development of numerical methods to approximate the mild solutions predicted by Theorems 5 and 6.

Author Contributions: Conceptualization, A.D.; methodology, D.F.M.T.; validation, A.D.; formal analysis, D.F.M.T.; investigation, D.F.M.T.; writing—original draft preparation, K.K.; writing-review and editing, A.D. and D.F.M.T.; supervision, A.D.; project administration, D.F.M.T. All authors have read and agreed to the published version of the manuscript.

Funding: Amar Debbouche and Delfim F. M. Torres were supported by FCT within the project UIDB/04106/2020 (CIDMA).

Conflicts of Interest: The authors declare no conflict of interest. The funder had no role in the design of the study; in the collection, analyses, or interpretation of data; in the writing of the manuscript, or in the decision to publish the results.

\section{References}

1. Samko, S.G.; Kilbas, A.A.; Marichev O.I. Fractional Integrals and Derivatives: Theory and Applications; Gordon and Breach: Amsterdam, The Netherlands, 1993.

2. Kilbas, A.A.; Srivastava, H.M.; Trujillo, J.J. Theory and Applications of Fractional Differential Equations; Elsevier Science Publicaion: Amsterdam, The Netherlands; Boston, MA, USA; Heidelberg, Germany, 2006.

3. Podlubny, I. Fractional Differential Equations; Academic Press: San Diego, CA, USA, 1999.

4. Diethelm, K. The Analysis of Fractional Differential Equations. An Application-Oriented Exposition Using Differential Operators of Caputo Type; Springer: Berlin/Heidelberg, Germany, 2010.

5. Almeida, R. Functional Differential Equations Involving the $\Psi$-Caputo Fractional Derivative. Fractal Fract. 2020, $4,29$.

6. Debbouche, A.; Fedorov, V.E. A Class of Fractional Degenerate Evolution Equations with Delay. Mathematics 2020, 8, 1700.

7. Nemati, S.; Torres, D.F.M. Application of Bernoulli Polynomials for Solving Variable-Order Fractional Optimal Control-Affine Problems. Axioms 2020, 9, 114.

8. Ahmed, H.M.; El-Borai, M.M.; El-Owaidy, H.M.; Ghanem, A.S. Impulsive Hilfer fractional derivative differential equations. Adv. Differ. Equ. 2018, 2018, 1-20.

9. Debbouche, A.; Antonov, V. Approximate controllability of semilinear Hilfer fractional differential inclusions with impulsive control inclusion conditions in Banach spaces. Chaos Solitons Fractals 2017, 102, 140-148.

10. Furati, K.M.; Kassim, M.D.; Tatar, N. Existence and uniqueness for a problem involving Hilfer fractional derivative. Comput. Math. Appl. 2012, 64, 1616-1626.

11. Gou, H.; Li, B. Existence of mild solutions for Sobolev-type Hilfer fractional evolution equations with boundary conditions. Bound. Value Probl. 2018, 2018, 48.

12. Gu, H.; Trujillo, J.J. Existence of mild solution for evolution equation with Hilfer fractional derivative. Appl. Math. Comput. 2015, $257,344-354$.

13. Harrat, A.; Nieto, J.J.; Debbouche, A. Solvability and optimal controls of impulsive Hilfer fractional delay evolution inclusions with Clarke subdifferential. J. Comput. Appl. Math. 2018, 344, 725-737.

14. Kavitha, K.; Vijayakumar, V.; Udhayakumar, R.; Nisar, K.S. Results on the existence of Hilfer fractional neutral evolution equations with infinite delay via measures of noncompactness. Math. Methods Appl. Sci. 2020, 44, 1438-1455.

15. Jaiswal, A.; Bahuguna, D. Hilfer fractional derivative Differential Equations with Almost Sectorial Operators. Differ. Equ. Dyn. Syst. 2020, 1-17, doi:10.1007/s12591-020-00514-y.

16. Mali, A.D.; Kucche, K.D. Nonlocal boundary value problem for generalized Hilfer implicit fractional differential equations. Math Methods Appl. Sci. 2020, 43, 8608-8631.

17. Subbaiyan, S.; Debbouche, A.; Wang, J.R. Approximate controllability of Hilfer fractional Sobolev type integrodifferential inclusions with nonlocal conditions. Int. J. Dyn. Syst. Differ. Equ. 2020, 10, 59-80.

18. Zhang, L.; Zhou, Y. Fractional Cauchy problems with almost sectorial operators. Appl. Math. Comput. 2015, $257,145-157$.

19. Zhou, Y. Basic Theory of Fractional Differential Equations; World Scientific: Singapore, 2014.

20. Pazy, A. Semigroup of Linear Operators and Applications to Partial Differential Equations; Applied Mathematical Sciences; Springer: Berlin, Germany, 1983; Volume 44. 